\title{
Controlling the Virus Output Via Urokinase Receptor and Integrin Signaling Guido Poli*‡
}

Address: AIDS Immunophatogenesis Unit \& Vita-Salute San Raffaele University and Scientific Institute, Via Olgettina n. 58, 20132 Milan, Italy

Email: Guido Poli* - poli.guido@hsr.it

* Corresponding author $\ddagger$ Presenting author

from 2005 International Meeting of The Institute of Human Virology

Baltimore, USA, 29 August - 2 September 2005

Published: 8 December 2005

Retrovirology 2005, 2(Suppl I):S76 doi:I0.II86/I742-4690-2-SI-S76

We have described that either urokinase-type plasminogen activator (UPA) inhibits HIV expression in monocytic U937 and U1 cell lines (M. Alfano et al., PNAS, 2002, 99:8862-67). We have observed that uPA inhibited HIV expression exclusively when both UPAR and CD18/ CD11b (Mac-1) were co-expressed at the cell surface. A second interactor of UPAR, FPRL1, was abundantly expressed on the surface of both unstimulated and stimulated U1 cells; however, peptide antagonists of FPRL1 did not interfere with HIV expression from U1 cells. Incubation of U1 cells with Trojan peptides expressing RhoA domains reversed the anti-HIV activity of uPA. In addition to cell line infection, uPA inhibited in vitro infection of primary monocyte-derived macrophages and virus replication from monocytes of infected individuals cultivated ex-vivo. Thus, RhoA-dependent cytoskeleton rearrangement and intracellular vesicles formation may be related to virion budding and entrapment in intracytoplasmic vacuoles. This is the first report linking integrin activation to a negative control of HIV replication, at least in monocyte/macrophages. 